\title{
Formazione e-learning degli insegnanti e pensiero creativo*
}

\section{Gaetano Domenici - Valeria Biasi - Anna Maria Ciraci}

Università degli Studi «Roma Tre», Dipartimento di Scienze della Formazione

doi: 10.7358/ecps-2014-010-dome

gaetano.domenici@uniroma3.it

valeria.biasci@uniroma3.it

annamaria.ciraci@uniroma3.it

\section{E-LEARNING TEACHER'S TRAINING AND CREATIVE THINKING}

\begin{abstract}
This paper deals with the complex theme of assessing teachers' professional competencies. It illustrates an empirical study conducted in 2012/2013 by Domenici, Biasi and Ciraci, for the Department of Education of "Roma Tre» University. The study involved 287 inservice primary school teachers in the Lazio region who graduated in Education through "Roma Tre» University's e-learning platform. It aimed to assess whether the distancelearning degree course the teachers had completed had increased their professional skills and in what directions. We used a specific on-line instrument, the CDVR questionnaire, in order to evaluate their teaching and relational competencies. The results show that the above e-learning university courses, conducted through an active didactics based on selfassessment and on innovative strategies for simulation environments, produced an increase in the teacher's perception of educational and evaluative skills, and in the teacher's perception of the ability to understand others, together with a greater mental flexibility, which means creativity. These active teaching methods, thanks to new technologies, have promoted the development of attitudes underlying the expert teacher, who is mentally flexible and creative in finding novel solutions to many educational problems. A better understanding of the challenges along with improved communication skills (thanks to better teaching and evaluation strategies and more in-depth psychological knowledge) led to a special effect on
\end{abstract}

* Questo articolo è il risultato del lavoro congiunto dei tre autori. In particolare G. Domenici ha scritto il par. 1; V. Biasi i parr. 2.1, 3.1, 3.2.1, 4; A.M. Ciraci i parr. 2.2, 2.3, 2.3.1, 3.2.2. 
the ability to interact and develop a positive attitude in the context of the educational relationship, a functional attitude to enhance the learning process and thus the achievement of the learning outcomes pursued.

Keywords: Creativity, Didactics competencies, Evaluation, Relational competencies, Teacher e-learning training.

\section{INTRODUZIONE: MIGLIORARE LA QUALITÀ DELLA FORMAZIONE DEGLI INSEGNANTI}

La qualità dell'insegnamento comincia ad essere finalmente considerata come una variabile altamente esplicativa della produttività culturale, dell'assetto motivazionale e disposizionale - in termini di apprendimento prodotto negli allievi - dei processi formativi formali e non-formali. Non a caso essa è considerata uno dei fattori chiave che determinerà nel prossimo futuro la misura in cui l'Unione Europea potrà incrementare la sua competitività in un mondo globalizzato. Come evidenziato da diverse ricerche sussiste una stretta correlazione fra la qualità professionale degli insegnanti e i risultati degli alunni (Darling-Hammond et al., 2005; Rivkin, Hanushek, \& Kain, 2005). Purtroppo quasi tutti i Paesi dell'Unione Europea (e non solo) segnalano lacune a livello delle competenze professionali degli insegnanti e indicano difficoltà nell'aggiornamento e nella formazione. Le lacune si riferiscono in particolare alla mancanza delle competenze necessarie per affrontare la nuova evoluzione dell' istruzione (ivi compreso l'apprendimento individualizzato, la preparazione degli alunni all'apprendimento autonomo, la capacità di gestire gruppi eterogenei anche per provenienza culturale, la preparazione dei discenti all'impiego ottimale delle TIC, ecc.). Già l'indagine dell'OCSE, Teachers matter. Attracting, developing and retaining effective teachers, del 2005, frutto di due anni di ricerche sulle politiche dell'istruzione e della formazione condotte in 25 paesi, inclusa l'Italia, aveva rilevato in quasi tutti i paesi dell'Unione Europea carenze e lacune nella preparazione degli insegnanti e difficoltà e mancanza di incentivi nell'aggiornamento e nella formazione (OECD, 2005). Difficoltà confermate dalle recenti indagini TALIS (Teaching and learning international survey), pilotate dall'OCSE e svolte su insegnanti e presidi (o direttori) della scuola media (insegnamento secondario di primo grado), che hanno messo in evidenza come, in molti sistemi scolastici, si spenda molto per la formazione continua degli insegnanti ma che gli esiti di questa formazione non sono quasi mai valutati e permane l'insoddisfazione, 
sia tra i partecipanti ai corsi sia tra le autorità che li finanziano; gli insegnanti hanno pochi incentivi a migliorare il loro insegnamento e le più comuni attività di sviluppo professionale a loro disposizione non sono le più efficaci, anche se la maggioranza degli insegnanti aspirerebbe a un maggiore sviluppo professionale (OECD, 2009 e 2014).

Nel 2007, nella Comunicazione al Consiglio e al Parlamento Europeo, Migliorare la qualità della formazione degli insegnanti, nell'ambito del Programma di lavoro istruzione e formazione 2010, la Commissione Europea pone un importante punto fermo nel percorso di costruzione di indicatori $e$ parametri di riferimento a proposito del profilo culturale e professionale di un buon insegnante e sulle competenze che devono costituire il bagaglio di ciascun individuo nella società della conoscenza: gli insegnanti dovrebbero essere in grado di appropriarsi in maniera significativa dei risultati della ricerca in ambito educativo e di far evolvere i propri saperi e le proprie competenze in funzione delle innovazioni, tenere il passo con i settori della conoscenza in rapido cambiamento. Essi dovrebbero altresì sviluppare competenze chiave, legate agli aspetti socio-culturali della società della conoscenza: gestire un'ampia gamma di conoscenze, saper utilizzare e integrare nella didattica le tecnologie, instaurare rapporti di fiducia con gli alunni, collaborare efficacemente con i colleghi e gli altri partner del settore educativo, incoraggiare il rispetto e la comprensione interculturale, padroneggiare i sempre nuovi approcci in tema di valutazione (Commissione Europea, 2007).

Per affrontare le aspettative e le sfide di una società sempre più globalizzata, sempre più mutevole, sempre più multiculturale, la qualità professionale dei docenti, ai quali è affidata l'educazione delle nuove generazioni, rappresenta, dunque, uno dei nodi centrali da sciogliere.

Il contributo qui presentato si colloca appunto nell'ambito della complessa tematica della individuazione, rilevazione e analisi delle competenze professionali degli insegnanti e della determinazione delle modalità didattico-formative più idonee per la loro acquisizione

Si tratta di alcuni esiti scaturiti da una ricerca empirica, condotta nel 2012/2013 da Domenici, Biasi e Ciraci, per il Dipartimento di Scienze della Formazione dell'Università «Roma Tre», che ha coinvolto 287 insegnanti in servizio nelle scuole primarie della Regione Lazio laureatesi presso il "Corso di Laurea in Scienze dell'Educazione in modalità FAD su piattaforma e-learning», dell'Università "Roma Tre». Tale indagine è stata orientata a verificare se, al termine del percorso formativo, ovvero dopo la laurea, vi sia stata una effettiva ricaduta, e, in caso positivo, di quale peso, in termini di incremento delle reali competenze professionali negli insegnanti laureati in modalità e-learning, rispetto a chi non ha avuto alcuna formazione universitaria. Al fine di rilevare tali ricadute è stato appositamente allestito uno specifico stru- 
mento, il Questionario CDVR (Competenze Didattico-Valutative e Relazionali), per la valutazione delle competenze didattico-valutative e relazionali dei docenti della scuola primaria e secondaria (Domenici, Biasi, \& Ciraci, 2014). Il suddetto questionario ha permesso l'individuazione degli aspetti da privilegiare e intorno a cui operare al fine di adattare il percorso formativo universitario e-learning il più possibile alle necessità concrete dell'agire professionale dei docenti e, in generale, ha promosso una riflessione critica, nella progettazione della formazione universitaria degli insegnanti, sulle competenze da favorire e sulle strategie didattiche più adeguate per svilupparle.

Il percorso formativo universitario e-learning dedicato agli insegnanti realizzato all'Università «Roma Tre», è particolarmente orientato a promuovere specifiche conoscenze metodologico-didattiche e psicologiche relative ai processi educativi e alle dinamiche dell'apprendimento, attraverso modalità didattiche attive che, grazie alle nuove tecnologie, non si limitano alla semplice erogazione di contenuti, ma mirano a far applicare le conoscenze in contesti, reali o simulati, al fine di trovare soluzioni adeguate ai problemi posti, promuovono lo sviluppo di atteggiamenti che sono alla base delle "competenze professionali» dell'insegnante esperto, capace di essere mentalmente flessibile, e quindi creativo, nel trovare soluzioni originali ai molteplici problemi educativi che si trova volta a volta ad affrontare.

Questa nuova modalità di formazione e-learning così strutturata ha prodotto, come vedremo, la percezione di un cambiamento nello stile relazionale adottato, sviluppando nei docenti così formati la percezione della capacità di comprendere gli altri e la flessibilità mentale, vale a dire la creatività (o capacità di problem solving). Il pensiero creativo - anche sulla base degli esiti qui indicati - appare caratterizzato dalla abilità di trovare molteplici soluzioni diverse a problemi che ammettono più soluzioni e rappresenta una risorsa nelle strategie di problem solving. Questo atteggiamento si lega anche alla percezione di avere più risorse per fronteggiare le difficoltà professionali.

Conoscere meglio chi si ha di fronte e comunicare meglio con chi si ha di fronte (grazie a migliori strategie didattico-valutative e a più approfondite conoscenze psicologiche), infatti, porta, come di seguito illustrato, ad una speciale ricaduta sulla capacità di interagire e di sviluppare un atteggiamento positivo nell'ambito della relazione educativa, atteggiamento funzionale al potenziamento dei processi di apprendimento e quindi al raggiungimento degli esiti formativi perseguiti. 


\section{PENSIERO CREATIVO E FORMAZIONE DEGLI INSEGNANTI}

\subsection{Il pensiero creativo nella ricerca psicologica sperimentale: applicazioni nel settore della formazione}

La ricerca psicologica in tema di creatività ha registrato importanti sviluppi, come testimoniato da una vasta serie di contributi classici e recenti, compresi i contributi di sintesi e ricapitolazioni degli studi effettuati (Torrance, 1966; Guilford, 1967; Runco \& Albert, 1990; Csikszentmihalyi, 1997; Krampen, 1997; Runco \& Richards, 1997; Sternberg, 1999; Andreani Dentici, 2001; Legrenzi, 2005). Gli studi hanno preso numerosi orientamenti, tra cui si annoverano quelli che seguono un approccio dinamico, che occupano un ruolo di rilievo in ambito metodologico (come riportato per esempio da Sternberg \& Lubart, 1999).

$\mathrm{Vi}$ sono inoltre contributi teorici e di osservazione sistematica centrati sul ruolo di alcuni tratti della personalità (Eysenck, 1993; Simonton, 1997), interessanti contributi sono forniti anche mediante la registrazione e l'analisi a breve termine, come quelli attivati da determinate condizioni ambientali, da specifiche istruzioni o prescrizioni. Queste condizioni possono produrre effetti diversi a seconda del tipo di personalità del soggetto coinvolto (Hinton, 1971; Leith, 1972; Falat, 2000), ma possono anche avere una validità più generale, come vedremo di seguito.

Sulla base dei dati ottenuti attraverso varie prove sperimentali, Bonaiuto, Biasi, Giannini, Bonaiuto, \& Bartoli, 1992, Bonaiuto, Giannini, \& Biasi, (2003), Biasi \& Bonaiuto, 2004, hanno potuto sviluppare un modello teorico generale sulle dinamiche di stress e di comfort.

Secondo questo modello psicodinamico, lo stress implica un sovraccarico di conflitto con conseguenze sulla sfera emotiva, motivazionale, e cognitiva.

Entro certi limiti, al fine di evitare e/o ridurre le caratteristiche sgradevoli di un tale attivazione affettiva, le persone possono respingere altri tipi di conflitto, tra i quali vi sono incongruenze cognitive e incertezze. Comfort e relax agiscono in modo contrario. Altri processi possono interferire, in particolare i meccanismi di difesa. Ad esempio, lo stress può aumentare le proiezioni di aggressione o può favorire sentimenti di inferiorità e di insufficienza. Dobbiamo prendere in considerazione questi processi anche se vogliamo comprendere e prevedere gli effetti finali sul comportamento.

In accordo con la letteratura psicodinamica e con rilevanti evidenze riportate dagli studi di psicologia generale, il conflitto psichico è un meccanismo centrale collegato alle situazioni di stress. Manipolando il livello di 
conflitto emotivo e cognitivo (per esempio, attraverso la gestione di trattamenti di stress o di comfort a breve termine) si possono attivare direttamente le principali emozioni, alcune motivazioni e impressioni riguardanti il sé e l'ambiente.

Entrando nel dettaglio, dimostrazioni sperimentali suggeriscono che vivere in uno stato di stress porta ad un aumento di conflitto emotivo e cognitivo. Di conseguenza si favorisce un aumento di ansia, rabbia, tristezza, vergogna, imbarazzo, senso di colpa, dolore e altre emozioni "negative», così come si attivano l'aggressività, il desiderio di movimento del corpo e la necessità di congruenza cognitiva. È stato rilevato in tali circostanze anche un aumento dei sentimenti di minaccia e insicurezza, impressioni di inferiorità e di insufficienza del sé.

Attraverso vari esperimenti è stato dimostrato che quando viene attivato questo assetto emotivo e motivazionale, vengono influenzati alcuni processi cognitivi secondo modalità prevedibili (Biasi, 2006). Ad esempio, i soggetti in condizioni di stress sperimentale a breve termine, sviluppano una intolleranza diffusa verso le incongruenze, le incertezze e le varie forme di ambiguità; un aumento dei processi che portano verso un incremento dell'attribuzione di significato, in particolare dell'attribuzione di causalità, aumenta cioè il bisogno di spiegazioni al fine di ridurre o prevenire le incongruenze che non sono altro che ulteriori contenuti conflittuali (Bonaiuto et al., 1992).

Tutti questi effetti cognitivi sembrano essere strettamente connessi con il tentativo di prevenire o ridurre il livello di conflitto psichico individuale esperito.

Come già accennato, un sovraccarico di conflitto favorisce l'intolleranza dell'incongruenza, dell'incertezza e dell'ambiguità - in quanto gli elementi fortemente incongrui, incerti e/o ambigui rappresentano in sostanza ulteriori conflitti. Si è dimostrato sperimentalmente che un aumento nell'attribuzione di causalità gioca facilmente un ruolo nel ridurre o evitare la percezione di incongruità, fornire cioè un rapporto co-determinazione significa fornire la spiegazione di fenomeni che altrimenti risulterebbero incomprensibili al soggetto.

Inoltre, Biasi e Bonaiuto (2004, 2007 e 2011) hanno dimostrato l'effetto a breve termine dell'attivazione di conflitto psichico nel campo della creatività: nel senso della sua inibizione a favore di un effetto di stereotipia. Ciò in quanto la creatività (per definizione) ha a che fare con l'accettazione di idee e figure nuove, quindi incongrue, strane, insolite e bizzarre, le quali sfidano e contraddicono gli schemi mentali precedentemente consolidati.

Questa evidenza sperimentale che deriva dalle indagini condotte nell'ambito della psicologia generale può rappresentare una buona base teorica per le applicazioni nel settore della formazione. 
A tale scopo intendiamo sottolineare l'importanza dello sviluppo della creatività nei percorsi di formazione degli insegnanti in particolare condotti in modalità e-learning attraverso una didattica attiva basata sull'auto-valutazione e su innovative strategie di simulazione di contesti, ciò al fine di ampliare le possibilità del docente di sviluppare strategie creative di problem solving, utili a fronteggiare ed eventualmente risolvere, le diverse e sempre più complesse questioni educative.

In quest'ottica, una maggiore comprensione dell'altro, e una migliore comunicazione (grazie a strategie didattico-valutazione avanzate e ad una più profonda conoscenza dei principi psicologici, come evidenzieremo nel corso del presente articolo), porta ad un effetto speciale sulla capacità di interagire e sviluppare un atteggiamento positivo all'interno della relazione educativa.

Questo atteggiamento produce a nostro avviso un miglioramento dei processi motivazionali e di apprendimento, e quindi può facilitare il raggiungimento dei risultati formativi perseguiti.

Tale cambiamento di atteggiamento si basa sul rafforzamento della capacità empatica e, in particolare, sullo sviluppo della flessibilità mentale, o creatività, al fine di far fronte alle frustrazioni (si vedano Hinton, 1971; Falat, 2000; Shahenn, 2010) e può fornire all'insegnante maggiori risorse per affrontare i vari problemi della professione e, quindi, prevenire lo sviluppo di modalità rigide e difensive.

Il rafforzamento delle competenze didattico-valutative e relazionali è volto anche, a livello psicologico, alla riduzione delle impressioni di impotenza e di stress, a vantaggio di un atteggiamento ricettivo e flessibile, orientato al problem solving.

\subsection{Il pensiero creativo in educazione: competenze degli insegnanti e creatività nei processi di insegnamento-apprendimento}

Da diversi anni nella letteratura internazionale (Argyris \& Schön, 1978; Schön, 1983; Carr \& Kemmis, 1986; Van Manen, 1993; Zeichner \& Liston, 1996; Parker, 1997; Ghaye \& Ghaye, 1998; Sternberg,1999; Furlong, Barton, Miles, Whiting, \& Whitty, 2000; Korthagen, 2001; Cesa-Bianchi \& Antonietti, 2003; Mortari, 2003; Clarke \& Chambers, 2005) si sottolineano, e soprattutto si cercano di indagare, gli aspetti creativi nell'ambito delle competenze degli insegnanti. Già John Dewey descriveva il processo riflessivo come una funzione fondamentale della mente stimolata da un "disagio cognitivo", uno stato di incertezza che richiede l'esame retrospettivo della situazione e il superamento del dubbio attraverso la strutturazione di nuove decisioni i cui esiti in quanto procedurali, producono un nuovo equilibrio in 
vista di nuovi problemi (Dewey, 1933). È proprio il venire a capo di questo senso di disorientamento e contenere l'incertezza che rappresenta la funzione biologica della cosiddetta creatività secondo l'ipotesi di Emilio Garroni (Garroni, 2010, p. 52) (solo per citare uno dei tanti filosofi, da Platone a Chomsky, che si sono interessati del problema) che, nel panorama degli ultimi tre decenni inflazionato da usi anche grotteschi del termine, offre una interpretazione rigorosa e puntuale su cosa sia il comportamento creativo. Secondo Garroni, il termine "creatività» assume significato se lo si correla al modo in cui la nostra specie si adatta all'ambiente, dunque al modo in cui essa esegue l'insieme dei compiti operativi e cognitivi che ne garantiscono la sopravvivenza. Opposta ad una ineffabile e romantica idea di innovazione scissa e contrapposta ad ogni sorta di regolarità o legalità, la creatività è, per Garroni, sulla scia di una lunga tradizione filosofica, teologica e giuridica, una procedura adattiva determinata dalla necessità e caratterizzata dalla elaborazione di soluzioni originali e non prefissate. Dunque identifica la creatività con l'attitudine a specificare leggi e principi generali. Essa sarebbe racchiusa, pertanto, nel modo in cui applichiamo una regola generale in un caso particolare, o specifichiamo una legge naturale in riferimento a certi fenomeni contingenti, o usiamo un principio intellettuale di portata universale in un'occasione irripetibile.

Ed è questa l'idea di creatività che possiamo ritrovare nella nuova epistemologia della pratica professionale riassunta nella proposta del "professionista riflessivo» (Schön, 1983; Mezirow, 1991). Si tratta di un'impostazione che cerca di capire come i professionisti affrontino quelle situazioni che sono impossibili da risolvere soltanto in termini tecnici, come ad esempio l'insegnamento, caratterizzate dalla necessità di agire in situazioni incerte, peculiari e nelle quali vi sono conflitti di valore. Questo processo costituisce, come la chiama Schön, la «riflessione nel corso dell'azione», ovvero la componente creativa nei professionisti del futuro.

Non è un caso che proprio nell'ambito scolastico le nuove istanze legate al pensiero riflessivo - suscitate dalla proposta di Schön, che è una proposta squisitamente educativa (tre dei casi progettuali analizzati da Schön sono casi "didattici») - si siano sviluppate molto celermente e abbiano dato vita a una rilevante mole di studi e sperimentazioni. Infatti insegnare vuol dire, spesso, "agire nell'urgenza, decidere nell'incertezza» e l'esercizio della competenza professionale passa attraverso operazioni mentali complesse, sottese da schemi di pensiero (Perrenoud, 1996), quelli che permettono di determinare (più o meno coscientemente e rapidamente) e di realizzare (più o meno efficacemente) un'azione relativamente adatta alla situazione.

Come dimostrato da un'ampia letteratura internazionale (Perrenoud, 2001; Domenici, 2009a; Altet, 2010), per gli insegnanti professionisti è in- 
dispensabile non già possedere un certo repertorio di tecniche e di linee precodificate di azione ma sviluppare, all'interno di una strategie di intervento flessibile, la capacità di cogliere il profilo originale delle situazioni contingenti e mettere in gioco quella "capacità diagnostica» che consente di «sentire» le situazioni fattuali, di differenziarle e modificarle. Sulla falsariga di Garroni si potrebbe dire che la creatività degli insegnanti ha il suo baricentro nella loro facoltà di giudizio (Garroni, 2010).

Se l'insegnante professionista deve essere un insegnante esperto in grado di riflettere sulle proprie pratiche per trovare nuove e appropriate tattiche per risolvere le situazioni problematiche che gli si presentano durante l'ordinaria situazione lavorativa, occorre far emergere expertise, intuizioni o "abilità artistiche», espressione della stretta interconnessione di teoria e pratica, che i professionisti sanno agire nelle situazioni di incertezza, unicità e conflitti di scelte oltre che di valore che quotidianamente si presentano loro. Sviluppare e valorizzare queste competenze negli insegnanti, trasformandole in un habitus professionale indispensabile per fronteggiare la dinamicità dei saperi e dei contesti, significa, però, andare ben oltre la mera esperienza.

La sola esperienza, infatti, non è elemento sufficiente, è necessaria una rielaborazione e una riflessione su di essa. Perché la conoscenza della pratica si trasformi in pensiero e professionalità consapevole occorre arrivare all'esplicitazione del significato di un'esperienza, alla sua reinterpretazione, per trasformarla in apprendimento intenzionale (Mezirow, 2003).

In questa prospettiva occorrono modalità didattiche attive che, mirando a far applicare le conoscenze in contesti, reali o simulati, al fine di trovare soluzioni adeguate ai problemi posti, possano promuovere lo sviluppo di atteggiamenti che, come abbiamo visto, sono alla base delle «competenze professionali» dell'insegnante esperto. E sono soprattutto le nuove tecnologie che possono permettere l'apprendimento "per esperienza». In un corso online l'«esperimento dimostrativo scolastico», infatti, diventa "esperienza diretta», grazie alle potenzialità dell'ipermedia che, utilizzando molteplici sistemi simbolici, aumenta l'efficacia dell'immagine con il coinvolgimento plurisensoriale e promuove il pensiero intuitivo, analogico, ramificato, pluriprospettico, concreto (Galliani, 2004). In una didattica online che non si limiti alla semplice erogazione di contenuti le tecnologie, purché adeguatamente situate ed integrate, "possono divenire risorse capaci di far emergere nuove forma di riflessività critica perché inducono in qualche modo a riflettere sulle regole sottese, sui criteri interni: la conquista di livelli di riflessione più alta (capacità di vedere i problemi secondo una pluralità di ottiche, di considerali secondo angolature inconsuete, consapevolezza dell'esistenza di relazioni più profonde, nascoste) rappresenta uno dei contributi più importanti che esse potranno fornire all'apprendimento"(Calvani, 2000). 


\subsection{Formare il docente creativo: le scelte didattiche del "Corso di Laurea in Scienze dell'Educazione in modalità FAD su piattaforma e-learning» dell'Università «Roma Tre»}

Le riflessioni appena compiute individuano alcuni elementi essenziali della più ampia prospettiva che caratterizza le modalità didattiche del "Corso di Laurea in Scienze dell'Educazione in modalità FAD su piattaforma elearning» di «Roma Tre», nato per la formazione degli insegnanti e scenario dell'indagine che qui viene presentata.

Il «Corso di Laurea in Scienze dell'Educazione in modalità FAD su piattaforma e-learning» dell'Università "Roma Tre» ${ }^{1}$ ha avuto origine dal desiderio dei suoi promotori di dar corso ad una formazione "in servizio" di livello universitario che, rispondendo significativamente alle aspettative e ai bisogni formativi dei docenti, potesse provocare una ricaduta positiva sui reali processi educativi scolastici e così promuovere un innalzamento della qualità dell'istruzione.

La scelta delle modalità didattico-organizzative è gioco forza caduta sull'e-learning, data la difficoltà, per studenti adulti e lavoratori, di conciliare la frequenza universitaria con gli impegni connessi alla professione di insegnante e all'organizzazione della vita personale e familiare.

Cardine dell'iniziativa è stata, dunque, la realizzazione di un'attività di formazione in servizio svolta prevalentemente attraverso una piattaforma e-learning, in grado di arricchire il lavoro educativo quotidiano senza generare interferenze con il suo pieno svolgimento, come accadrebbe, invece, con la frequenza solo «in presenza» delle attività universitarie. L'attività formativa si caratterizza, inoltre, per una organizzazione modulare di presentazione, di studio e di fruizione del materiale didattico impiegato per l'insegnamentoapprendimento. L'accesso alle attività didattiche online avviene, cioè, per moduli, ossia per aggregati di materie considerate altamente congruenti per i contenuti che le caratterizzano e/o per le metodologie che le contraddistinguono. In questo modo non solo si evitano eccessive ridondanze nelle questioni tematiche trattate, ma, la possibilità di sostenere contestualmente gli esami relativi all'intero modulo, permette una capitalizzazione delle risorse, sia nel momento dello studio che nel momento dell'esame finale (Domenici, 2009b).

1 Il «Corso di Laurea in Scienze dell'Educazione in modalità FAD su piattaforma e-learning", di cui è Responsabile scientifico Gaetano Domenici, è in atto dall'anno accademico 2003/2004. Gli iscritti al corso sono tutti insegnanti, con un'età media, al momento della immatricolazione, di circa 45 anni, in servizio nella scuola dell'infanzia e primaria della Regione Lazio, in massima parte donne. 
Però, in un corso di laurea rivolto a studenti adulti, lavoratori e insegnanti, non basta prevedere una flessibilità di spazi e di tempi maggiormente compatibile con i loro ritmi di lavoro e stili di apprendimento, ma occorre mirare ad una ricaduta professionale dei processi formativi attivati. L'adulto è, infatti, più disposto ad apprendere ciò che risulta concretamente utilizzabile ed efficace, seppur indirettamente, nelle situazioni della vita reale, rispetto a ciò che non lo è (Weiner, 1972; Demetrio, 1991; Bandura, 2000). Nel "Corso di Laurea in Scienze dell'Educazione in modalità FAD su piattaforma e-learning» dell'Università "Roma Tre» le modalità didattiche, grazie anche alle potenzialità della rete, si basano, quindi, più che sulla trasmissione di saperi precostituiti, su proposte di attività che si configurano come vere e proprie simulazioni di eventi situati nei contesti dell'insegnamento e da strumenti valutativi e autovalutativi.

In particolare le attività basate sulla simulazione di contesti incoraggiano chi vi si impegna nell'applicare talvolta un vero e proprio transfert di apprendimento e, in genere, una cosiddetta «ristrutturazione degli elementi del campo» (Köhler, 1925): attraverso questa operazione mentale diviene possibile modificare il ruolo in cui è percepito o comunque colto un elemento all'interno del contesto, ha importanza dunque la capacità di mettere le parti importanti in relazione per coglierne il valore funzionale, trasformando la situazione.

In modo altrettanto fecondo, la procedura di autocontrollo si avvale di prove semistrutturate (Domenici, 1991, 2001 e 2005), che prestano attenzione tanto ai processi quanto ai risultati, al come si apprende oltre che al cosa e che, attraverso simulazioni di contesti reali, facendo riferimento a problemi complessi e incerti come quelli che si incontrano normalmente nella vita di tutti i giorni, permettono di sviluppare la consapevolezza dei propri processi cognitivi e la capacità di porre in essere strategie per risolvere un problema (Ciraci, 2009 e 2014; Domenici, 2009b).

Nell'ambito di una proposta formativa e-learning di matrice costruttivista, i processi di autovalutazione, soprattutto attraverso la simulazione di contesti reali, permettendo allo studente di prendere decisioni, di osservarne le conseguenze e di riflettere sugli esiti sbagliati o incompleti, favoriscono la presa di coscienza delle proprie strategie di utilizzazione dei saperi posseduti, delle procedure valutative poste in essere e delle decisioni adottate nella soluzione di un problema. L'autovalutazione, quindi, se svolta in modo da favorire la capacità individuale di prevedere, pianificare, monitorare, valutare in modo sistematico la propria attività cognitiva, rappresentando una leva fondamentale per acquisire una piena consapevolezza delle proprie strategie cognitive, relazionali e affettivo-motivazionali, può costituire, nei processi formativi e-learning, uno dei fattori cruciali per migliorare i livelli di apprendimento degli allievi. 
Inevitabilmente, dalla dimensione metacognitiva nell'acquisizione delle conoscenze il discorso non può non spostarsi sul significato stesso di «competenza» (Perrenoud, 1998; Domenici, 2000 e 2009a; Le Boterf, 2000; Ajello, 2002; Rey, Carette, Defrance, \& Kahn, 2003; Ciraci \& Capogna, 2005; Maccario, 2006; Deci \& Moller, 2007; Baldacci, 2010; Pellerey, 2010 e 2011).

Le competenze, infatti, pur se indirizzate verso l'azione, superano i saper fare, non si limitano a spostare le conoscenze dal luogo della loro costruzione a quello della loro utilizzazione, perché implicano la cognizione delle ragioni dell'operare e la capacità di autoregolazione dell'azione (Ciraci, 2013). Per questo non bastano corsi di formazione per gli insegnanti che «spieghino» le teorie, ricadendo in una mera «trasmissione» del sapere o che, semplicisticamente, forniscano una meccanica applicazione delle teorie nel contesto professionale.

Sviluppare una competenza significa prendere in considerazione i tipi di situazione che mette in grado di padroneggiare, le risorse che vengono messe in campo (conoscenze teoriche e metodologiche, abilità, saper fare specifici e schemi di comportamento) e l'essenza degli schemi di pensiero atti ad impegnare le capacità di azione in una situazione complessa (Perrenoud, 2001). Se le "competenze» si esprimono nella "capacità di adottare strutture, piani, schemi e programmi di azione capaci di integrare a livello interdisciplinare le conoscenze, formali e informali, teoriche, esperienziali e procedurali possedute per risolvere un problema in un contesto ambientale specifico; di adottare, inoltre, un sistema di monitoraggio della validità del programma nel contesto specifico (meta-cognizione), quindi di ri-adattarlo (meta-valutazione e meta-decisione), costruttivamente, per porre in atto comportamenti adatti al raggiungimento degli scopi, ovvero per il raggiungimento di un risultato adeguato alle intenzioni stabilite» (Domenici, 2000 e 2009a), se ciò che produce "competenza» è la capacità di applicare le conoscenze, di renderle produttive, è opportuno che tale capacità venga appresa insieme ai saperi stessi. Una competenza si stabilizza quando la mobilitazione delle conoscenze aziona schemi costituiti, perché sono gli schemi che ci permettono di mobilitare le conoscenze, i metodi, le informazioni, le regole per affrontare una situazione (Piaget, 1974). E gli schemi si costruiscono a seguito di una pratica costante, di allenamento (Perrenoud, 1998 e 2002) e si articolano in base sia alle esperienze sia alle esigenze, alle motivazioni del soggetto che percepisce ed apprende (Biasi, Chiappetta Cajola, \& Bonaiuto, 2010). Si tratta, in sostanza, di utilizzare nei processi formativi in modo costante e sistematico, strumenti valutativi e autovalutativi che prestino attenzione tanto ai processi quanto ai risultati, al come si apprende oltreché al cosa si apprende. L'«apprendere ad apprendere», che rappresenta il paradigma 
formativo delle attuali società complesse, della conoscenza, comporta, infatti, non solo il possesso di saperi specifici, ma anche la consapevolezza di saper gestire i propri processi di apprendimento.

Come si sa, l'acquisizione e la gestione, a un livello soddisfacente di padronanza, delle principali strategie cognitive, affettive e motivazionali coinvolte nell'apprendimento sono considerate oggi come le competenze essenziali per poter proseguire nei successivi percorsi di apprendimento (Pellerey, 1996).

$\mathrm{Si}$ sottolinea quindi che, attraverso una formazione condotta con le suddette modalità e-learning, si incoraggia lo sviluppo di atteggiamenti sottostanti le "competenze professionali» del docente esperto, che è capace di essere mentalmente flessibile e quindi creativo nella ricerca di soluzioni originali non solo ai molti problemi educativi che incontra quotidianamente, ma anche come contributo individuale a quelli che caratterizzano l'attuale contesto storico.

\subsubsection{Didattica in modalità e-learning vs didattica classica in presenza}

Le modalità didattiche attive appena indicate, basate essenzialmente su proposte di attività che si configurano come vere e proprie simulazioni di eventi situati nei contesti dell'insegnamento e da strumenti valutativi e autovalutativi, non solo hanno prodotto negli studenti/insegnanti del "Corso di Laurea in Scienze dell'Educazione in modalità FAD su piattaforma e-learning» un effetto di maturazione delle competenze professionali, con una effettiva ricaduta sugli esiti procedurali dell'insegnamento (Ciraci, 2014; Domenici, Biasi, \& Ciraci, 2014), ma anche un evidente miglioramento degli esiti del percorso universitario. Come risulta da diverse indagini (Domenici, Margottini, \& Cajola, 2006; Ciraci, 2008 e 2009; Domenici, 2009b; Margottini, 2009), gli studenti del "Corso di Laurea in Scienze dell'Educazione in modalità FAD su piattaforma e-learning» hanno migliori performance rispetto agli studenti del corrispondente "Corso di Laurea in Scienze dell'Educazione in presenza" dello stesso ateneo, sia in termini di crediti conseguiti per anno accademico, sia in termini di voto medio, sia in termini di riduzione degli abbandoni. Dall' analisi delle carriere degli studenti nei primi due anni accademici infatti, emerge che gli studenti del corso online conseguono risultati piuttosto buoni in termini di progressione negli studi. È interessante osservare l'andamento della curva di distribuzione dei crediti nei primi due anni che si presenta a J per gli studenti FAD, mentre risulta rovesciata per gli studenti dei corsi tradizionali, con un numero elevato di studenti che presenta un forte ritardo negli studi e un numero ridotto di studenti in regola con i tempi previsti dal piano di studi (Figura 1). 
Numero di crediti conseguiti dopo due anni - Confronto studenti SdE - Sde-FAD

\begin{tabular}{|c|c|c|c|c|}
\hline Crediti & Num. Stud. FAD & $\%$ Stud. FAD & Num. Stud. SdE & $\%$ Stud. SdE \\
\hline 1 a 20 & 49 & 5,6 & 28 & 22,3 \\
\hline 21 a 40 & 53 & 6 & 29 & 23 \\
\hline 41 a 60 & 155 & 17,7 & 16 & 20,6 \\
\hline 61 a 80 & 160 & 18,2 & 16 & 12,7 \\
\hline 81 a 100 & 343 & 39,1 & 21 & 16,7 \\
\hline 101 a 120 & 117 & 13,3 & 6 & 4,8 \\
\hline
\end{tabular}

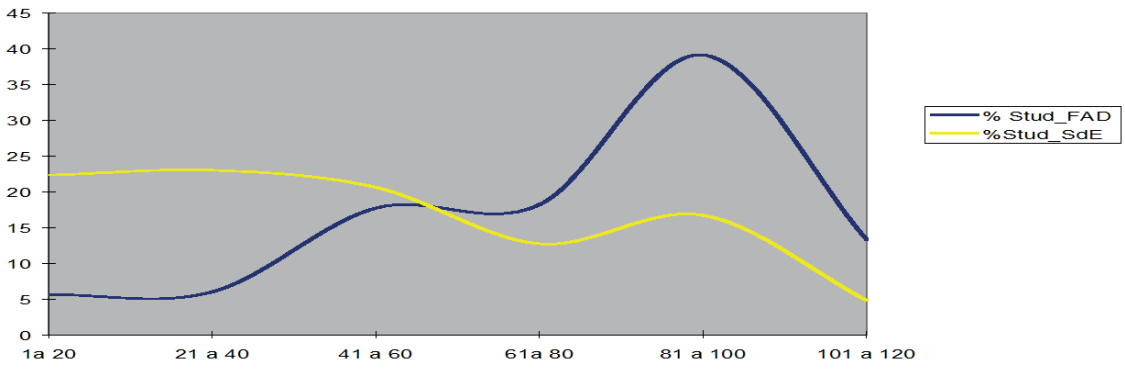

Figura 1. - Crediti conseguiti dagli studenti del corso online e dagli studenti del corso in presenza.

Nello stesso tempo, la ricerca svolta nell'ambito del progetto PRIN 20062008 (Domenici, 2009b), che si è avvalsa del "Corso di Laurea in Scienze dell'Educazione in modalità FAD su piattaforma e-learning» dell'Università "Roma Tre», ha mostrato a sua volta che il maggior utilizzo degli strumenti di autovalutazione a disposizione si associava a performance migliori (in termini di media dei voti, numero di esami e numero di crediti), confermando l'idea che l'autovalutazione, soprattutto se strutturata come simulazione di contesti, rappresenti una variabile cruciale per acquisire in modo significativo, stabile ed efficace quanto propone la formazione. I dati hanno evidenziato, infatti, in modo chiaro l'esistenza di una correlazione positiva tra sistematici processi autovalutativi delle competenze acquisite attraverso strumenti idonei allo scopo (prove semistrutturate) da una parte e performance (media dei voti, numero di esami e numero di crediti) dall'altra. Dall'analisi del campione totale dei casi osservati emerge un significativo legame diretto tra l'autovalutazione, le esercitazioni ed $i$ post alle esercitazioni da una parte e le performance dall'altra (Ciraci, 2009). L'indagine suddetta ha mostrato che, in un processo formativo e-learning, l'uso numeroso e sistematico degli strumenti di autovalutazione basati su compiti autentici, che diano enfasi alla 
costruzione della conoscenza e non alla sua riproduzione, che rappresentino la naturale complessità del mondo reale, favorendo la consapevolezza della propria situazione di apprendimento e delle strategie da usare in relazione ai propri limiti, alle proprie risorse e all'obiettivo da conseguire, possono migliorare i livelli di apprendimento degli studenti e i risultati della formazione in generale.

\section{UN'INDAGINE EMPIRICA PER LA RILEVAZIONE}

DELLE COMPETENZE DIDATTICO-VALUTATIVE E RELAZIONALI DEL DOCENTE NELL'OTTICA DELLO SVILUPPO DELLA CREATIVITÀ NEI PROCESSI DI INSEGNAMENTO

\subsection{Ipotesi}

Il percorso formativo universitario e-learning qui presentato, caratterizzato dalle modalità didattiche attive sopra citate e dedicato agli insegnanti, è particolarmente orientato a promuovere specifiche conoscenze metodologicodidattiche e psicologiche relative ai processi educativi e alle dinamiche dell'apprendimento.

$\mathrm{Ci}$ aspettiamo che tale percorso universitario triennale rinforzi nel docente un atteggiamento empatico, e sviluppi una maggiore capacità di problem solving creativo o flessibilità mentale, grazie al fatto che - conoscendo meglio la psicologia del discente e le diverse strategie didattiche e valutative -, il docente disponga di maggiori risorse per modulare il proprio stile educativo al fine di facilitare gli apprendimenti degli allievi.

Intendiamo rilevare questi cambiamenti attraverso la valutazione delle competenze didattiche, valutative e relazionali del docente, sviluppate grazie al suddetto training formativo universitario e-learning, e rilevate somministrando il Questionario CDVR, appositamente allestito in versione online.

\subsection{Metodologia, procedura, partecipanti, strumento e indicatori}

\subsubsection{Partecipanti}

Lindagine ha comportato l'allestimento e l'applicazione del Questionario CDVR (Competenze Didattiche, Valutative e Relazionali) di 287 docenti di ruolo in servizio nelle scuole primarie della Regione Lazio laureati presso il 
"Corso di Laurea in Scienze dell'Educazione su piattaforma e-learning» (immatricolatisi a partire dall'a.a. 2004/2005 e laureatisi entro gennaio 2012; con un'età media, al momento della immatricolazione, di circa 45 anni e in massima parte donne). Il questionario è stato compilato in modalità online.

Sono state inoltre tracciate comparazioni con un gruppo di controllo costituito da laureati, non impegnati professionalmente nella scuola, che hanno seguito lo stesso corso di laurea, con gli stessi docenti, ma con modalità didattica classica, cioè «in presenza».

\subsubsection{Strumento e indicatori. Il Questionario CDVR per la rilevazione delle competenze professionali didattiche, valutative e relazionali degli insegnanti}

Il difficile lavoro della identificazione delle competenze generali e specifiche degli insegnanti deve possedere opzioni teoriche e politico-culturali; per fare formazione occorre esplicitare il profilo professionale perseguito e per definire il profilo occorre avere un'idea sufficientemente precisa del modello di scuola considerato. Le competenze degli insegnanti vanno definite quindi all'interno di un profilo professionale esplicito e strutturato in termini di ciò che un individuo deve sapere e saper fare per essere un «bravo insegnante» nella scuola dell'autonomia.

Lo strumento CDVR per la rilevazione delle competenze professionali degli insegnanti utilizzato è stato elaborato tenendo presente un profilo professionale degli insegnanti rispondente ad alcuni orientamenti teorici internazionalmente riconosciuti, dei quali si riportano alcuni riferimenti:

1. Il primo è dato dalla proposta che emerge già dalle indagini OECD-CERI del 1994 e 1998, secondo le quali le competenze che contraddistinguono il «bravo insegnante» sono, fondamentalmente, le seguenti: le competenze metodologico-didattiche (incluse quelle valutative); le competenze disciplinari; le competenze comunicative e relazionali; le competenze organizzative (che riguardano anche l'insieme delle attività esterne alla classe); la «riflessività», cioè la capacità di riflettere criticamente sulla propria pratica professionale (OECD, 1994 e 1998).

2. Il secondo consiste nell'accogliere l'idea di una nuova epistemologia della pratica professionale, riassunta nella proposta del «professionista riflessivo» (Schön, 1983) ${ }^{2}$. Si tratta di un'impostazione che cerca di capire co-

2 La definizione originale utilizza il termine anglosassone practitioner, che in italiano è stato reso con professionista: occorre sottolineare che questo termine, in inglese, permette di far risaltare le componenti di creatività e maestria necessarie alla pratica professionale. 
me i professionisti affrontino quelle situazioni che sono impossibili da risolvere soltanto in termini tecnici, come ad esempio l'insegnamento, caratterizzate dalla necessità di agire in situazioni incerte, peculiari e nelle quali vi sono conflitti di valore.

3. Il terzo, sottolineato nella Comunicazione della Commissione al Parlamento Europeo e al Consiglio, Migliorare la qualità della formazione degli insegnanti, del 3 agosto 2007, riguarda la necessità per i docenti di lavorare con le più moderne tecnologie, di integrarle in modo efficace nell'insegnamento e nell'apprendimento, di essere in grado di orientare e sostenere le persone in apprendimento nei network in cui si può trovare e produrre informazione. Le tecnologie dell'informazione e della comunicazione possono infatti dare un importante contributo nel passaggio del nostro sistema formativo da un insegnamento basato sulle conoscenze a una didattica centrata sulle competenze e l'e-learning può rappresentare una forte spinta al rinnovamento delle pratiche formative, soprattutto di quelle rivolte allo sviluppo professionale dei docenti, verso l'adozione di metodologie attive, in grado di spostare l'attenzione sulla persona intesa come soggetto autonomo nell'imparare ad imparare, a scegliere, a relazionarsi.

4. Il quarto fa riferimento alla definizione ufficiale di competenza, contenuta nella Raccomandazione del Parlamento Europeo e del Consiglio del 23/04/2008 sulla costituzione del Quadro europeo delle qualifiche per l'apprendimento permanente, che considera competenza la "comprovata capacità di utilizzare conoscenze, abilità e capacità personali, sociali e/o metodologiche, in situazioni di lavoro o di studio e nello sviluppo professionale e personale. Nel contesto del Quadro europeo delle qualifiche le competenze sono descritte in termini di responsabilità e autonomia».

L'obiettivo è stato dunque quello di avviare una ricognizione su alcuni nuclei, da noi ritenuti fondanti, della professione insegnante, rappresentati da aree di attività, utilizzate nella pratica didattica quotidiana e a cui abbiamo riconosciuto una sostanziale capacità descrittiva della professione.

Il Questionario CDVR è costituito pertanto da 5 sezioni: (1) Dati ascrittivi e posizione lavorativa; (2) Strategie didattiche; (3) Strategie valutative; (4) Uso delle tecnologie; (5) Autoefficacia percepita; (6) Stile educativo e relazionale.

Il questionario, composto da domande ad alternative di risposta predeterminate, è stato auto compilato nel periodo ottobre/dicembre 2013 sul web (http://217.194.7.18/web/cati/UNIROMA3_FAD/inizializza.asp). 


\section{RISULTATI, COMMENTI E CONCLUSIONI}

I dati sono stati sottoposti al calcolo delle differenze tra le medie applicate a due percentuali di due campioni differenti. Si tratta di un test parametrico che studia la variabile differenza standardizzata delle percentuali. Questa si distribuisce come una variabile "Z" per cui per valori esterni superiori all'intervallo di fiducia considerato accettabile $(-1,96+1,96)$ si presume che la differenza registrata sia tale da ipotizzare che le due percentuali provengano da campioni di popolazioni differenti.

Si precisa che all'analisi delle diverse domande cosiddette "piene» cioè senza filtri, si nota che il numero dei rispondenti diminuisce leggermente, ma tale quota resta sempre sotto o intorno al $5 \%$ anche se si toccano punte oltre il $10 \%$ : ciò rende i risultati affidabili.

I risultati complessivi concernono la percezione di una maturazione delle competenze didattiche-valutative e relazionali, da parte dei docenti che hanno conseguito una formazione universitaria online condotta con strategie didattiche attive e flessibili.

In sintesi, da parte di tali docenti laureati, si sono registrati risultati quali-quantitativi rilevanti nella direzione di una crescita della percezione delle competenze didattico-valutative possedute (rispettivamente un elevato accrescimento nell' $85.0 \%$ dei casi per le competenze valutative; nel $75.3 \%$ dei casi per le competenze metodologico-didattiche). Si aggiunge la percezione di una maggiore capacità di utilizzare la documentazione educativa nel $76.0 \%$ dei casi, di progettare nel $60.5 \%$ dei casi, e di fare ricerca e sperimentazione nel $54.1 \%$ dei casi (Figure 2 e 3 ).

Si rileva inoltre una maggiore capacità relazionale, un cambiamento percepito, dopo l'esperienza universitaria, del proprio stile relazionale a seguito della formazione in Didattica (intensità massima percepita del cambiamento: punti 8 per il $29.7 \%$ dei docenti intervistati), in Docimologia (intensità massima percepita del cambiamento: punti 7 per il $27.0 \%$ dei docenti intervistati) ed in Psicologia (intensità massima percepita del cambiamento: punti 8 per il $22.9 \%$ dei docenti intervistati).

Inoltre, rispetto alla percezione di specifiche competenze, i docenti percepiscono un cambiamento dopo aver partecipato ai corsi online di Didattica (Figura 4), di Docimologia (Figura 5) e di Psicologia (Figura 6), i dati a questo proposito indicano incrementi in particolare rispetto alla "capacità di comprendere gli altri», allo «sviluppo della creatività» e del «pensiero critico».

Questo effetto sui punteggi di auto-percezione indica che gli insegnanti avvertono di aver sviluppato, a seguito del triennio di formazione universitaria online, specifiche competenze relazionali, sensibilità e atteggiamenti creativi, che si potranno rivelare utili per fronteggiare i diversi problemi educativi. 
II Corso di Laurea in Scienze dell'Educazione ha prodotto una ricaduta effettiva in termini di un accrescimento delle sue competenze professionali?

VALORI \%

$\square$ Valutazioni meno intense $\square$ Molto e moltissimo

Percezione di accrescimento delle competenze

\section{Organizzativo}

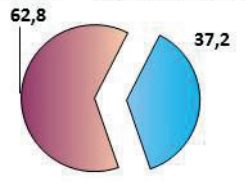

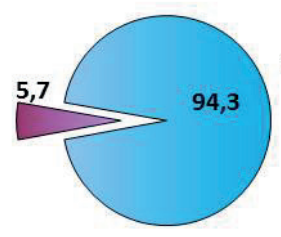

$\square$ No $\square$ Si

I66 Risp. Intervallo di confidenza $=+1-7,6 \%$

In quali campi?

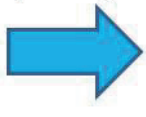

Tecnologicoed informatico

Metodologico

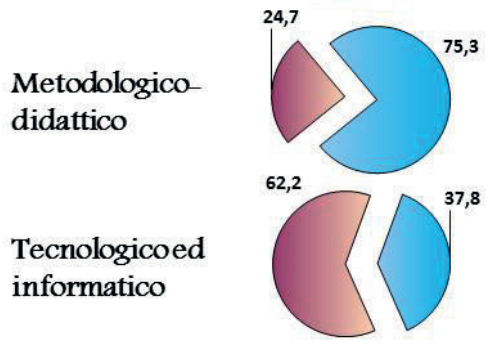

Documentazione

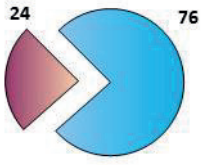

I53 Risp. Intervallo di confidenza $=+1-7,9 \%$

Figura 2. - Auto-percezione di incremento delle competenze organizzative, metodologico-didattiche, tecnologiche e della capacità di utilizzare la documentazione educativa, ottenuta da 287 docenti al Questionario CDVR.

II Corso di Laurea in Scienze dell'Educazione ha prodotto una ricaduta effettiva in termini di un accrescimento delle sue competenze professionali?

VALORI \%

$\square$ Valutazioni meno intense $\square$ Molto e moltissimo

Percezione di accrescimento delle competenze

\section{Progettazione}
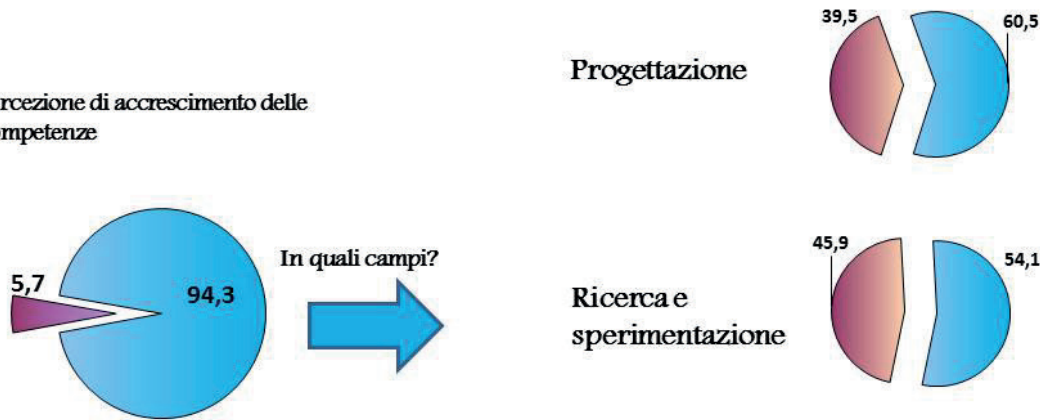

Ricercae sperimentazione

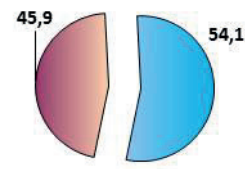

No $\square$ si

166 Risp. Intervallo di confidenza $=+\mid-7,6 \%$

Valutativo

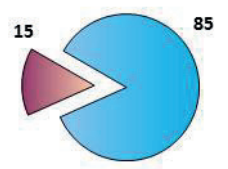

124 Risp. Intervallo di confidenza $=+1-8,8 \%$

Figura 3. - Auto-percezione di incremento delle competenze di progettazione, di ricerca e sperimentazione e di valutazione, ottenuta da 287 docenti al Questionario CDVR. 


\section{Cambiamento stile relazionale a seguito della formazione in Didattica}

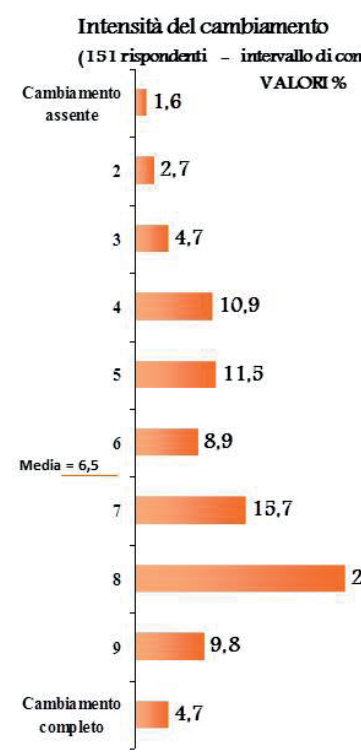

confidenza $=+1-8,0 \%$

Quali competenze si sono modificate a seguito del corso?
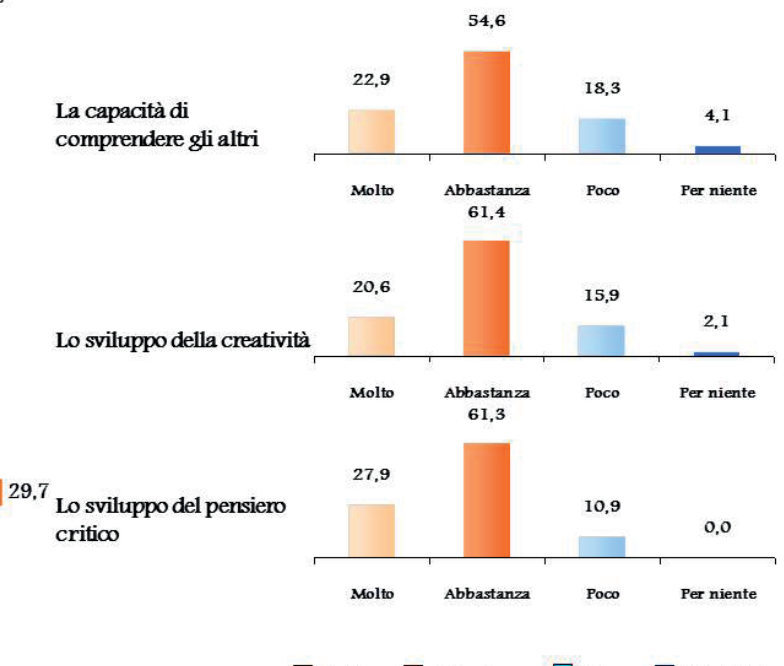

Scala da I (nessun cambiamento) a 10 (cambiamento completo)

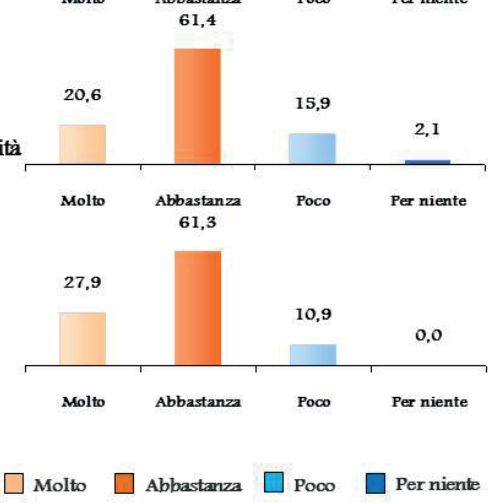

Figura 4. - Auto-percezione di incremento della capacità di comprendere gli altri, dello sviluppo della creatività e del pensiero critico, ottenuta a seguito della formazione in Didattica da 287 docenti al Questionario CDVR.

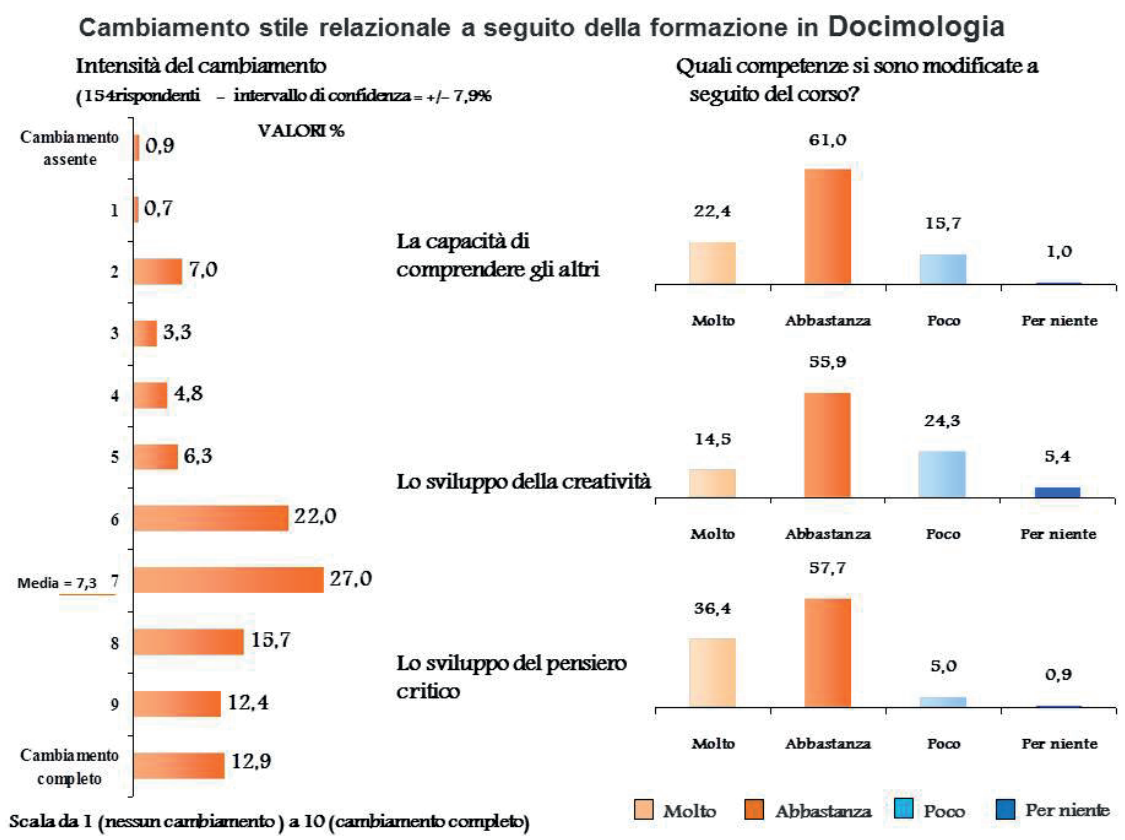

Figura 5. - Auto-percezione di incremento della capacità di comprendere gli altri, dello sviluppo della creatività e del pensiero critico, ottenuta a seguito della formazione in Docimologia da 287 docenti al Questionario CDVR. 


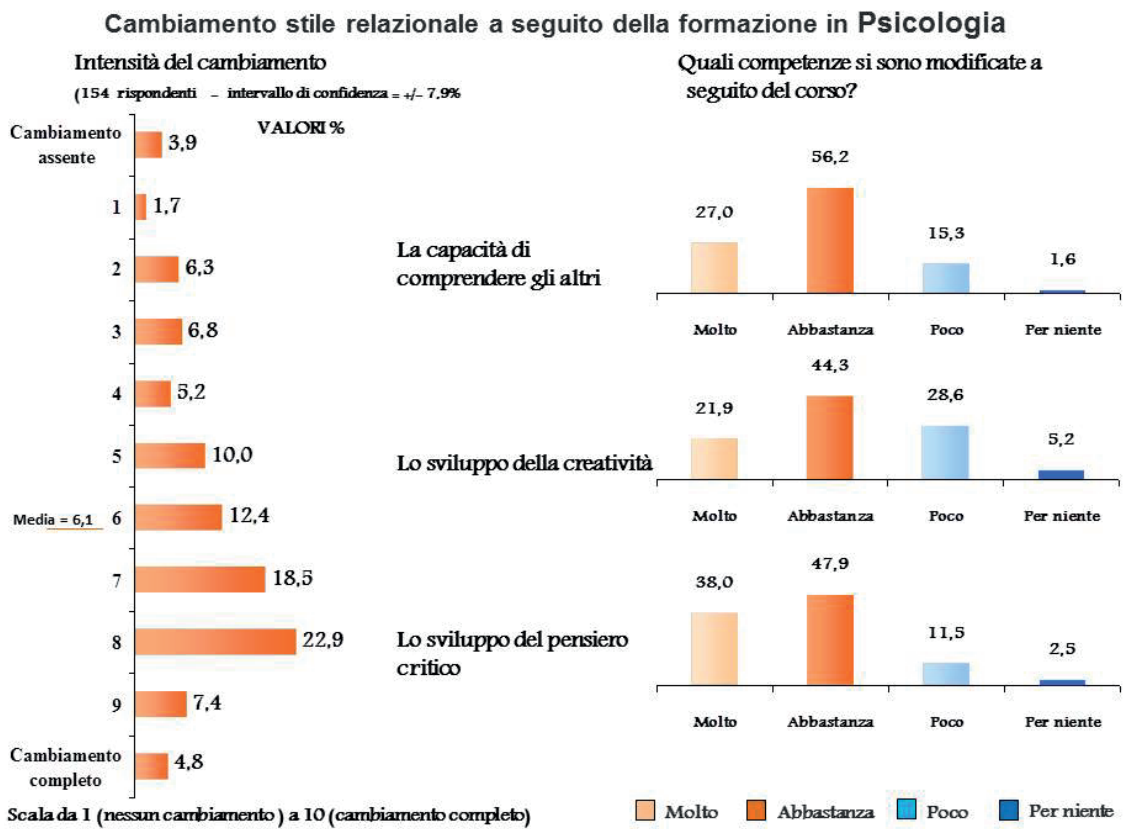

Figura 6. - Auto-percezione di incremento della capacità di comprendere gli altri, dello sviluppo della creatività e del pensiero critico, ottenuta a seguito della formazione in Psicologia da 287 docenti al Questionario CDVR.

In sintesi, i dati raccolti sottolineano non solo l'incremento delle competenze didattiche, valutative e relazionali a seguito del corso di formazione universitaria triennale, ma dimostrano come il suddetto percorso formativo universitario e-learning dedicato agli insegnanti, abbia prodotto a sua volta anche un cambiamento nello stile relazionale adottato.

A nostro avviso, questo tipo di formazione basata su modalità didattiche attive che, grazie alle tecnologie, non si limita alla semplice erogazione di contenuti, ma mira a far applicare le conoscenze in contesti, reali o simulati, al fine di trovare soluzioni adeguate ai problemi posti, promuove lo sviluppo di atteggiamenti che sono alla base delle "competenze professionali» dell'insegnante esperto, capace di essere mentalmente flessibile, e quindi creativo, nel trovare soluzioni originali ai molteplici problemi educativi che si trova ad affrontare.

Una ulteriore importante ricaduta della formazione universitaria triennale conseguita dal gruppo dei docenti intervistati, riguarda il cambiamento 
registrato nell'ambito dello stile relazionale adottato, in particolare per i seguenti indicatori - che mostrano un'evidente dissomiglianza dall'ipotesi di equidistribuzione -, dai quali si evince (cfr. Figure 7-9):

- una minore impulsività (la maggioranza dei docenti indica di reagire poco o per niente su base emotiva rispetto al passato);

- una minore insicurezza rilevata dal fatto che vi è una minore ricerca del riconoscimento sociale della propria attività;

- una maggiore capacità di decidere, ossia essere meno incerti nel prendere decisioni sempre rispetto al passato;

- una maggiore determinazione espressa nella capacità di portare a termine gli impegni, di cercare le ragioni delle difficoltà incontrate nella professione, di contrastare forme di ansia, di collegare le conoscenze già possedute alle nuove acquisizioni;

- una maggiore responsabilizzazione professionale nel senso di attribuire maggiormente la riuscita o il fallimento al proprio impegno personale, quindi una crescita del cosiddetto lucus of control interno che permette lo sviluppo di un atteggiamento positivo, costruttivo verso le difficoltà professionali e riduce in genere la percezione di impotenza e di stress;

- lo sviluppo, sempre nell'arco del triennio, di un atteggiamento più empatico (maggiore attenzione alle esigenze altrui) e pro-sociale ("cerco di aiutare gli altri») probabilmente legato anche al fatto di avere più risorse per fronteggiare le varie problematiche professionali.

In sintesi si sottolinea come la crescita delle competenze didattico-valutative e relazionali a seguito di questa formazione universitaria triennale abbia prodotto - non solo conoscenze specifiche di nuove strategie metodologicodidattiche e di specifici contenuti psicologici relativi ai processi educativi e alle dinamiche dell'apprendimento - ma che queste conoscenze hanno a loro volta hanno prodotto la percezione di un cambiamento del modo di porsi in relazione col discente, nel rispetto delle differenze interindividuali, facendo registrare un cambiamento nella qualità della relazione educativa.

In sintesi, questo percorso formativo universitario e-learning dedicato agli insegnanti, orientato a promuovere specifiche conoscenze metodologico-didattiche e psicologiche relative ai processi educativi e alle dinamiche dell'apprendimento, ha prodotto a sua volta anche un cambiamento nello stile relazionale adottato, sviluppando nel docente la percezione della capacità di comprendere gli altri e la flessibilità mentale, vale a dire la creatività (o capacità di problem solving). Il pensiero creativo è infatti caratterizzato dalla abilità di trovare molteplici soluzioni diverse a problemi che ammettono più soluzioni e rappresenta una risorsa nelle strategie di problem solving. Questo atteggiamento si lega alla percezione di avere più risorse per fronteggiare le difficoltà professionali. 


\section{Cambiamento stile relazionale nel triennio}

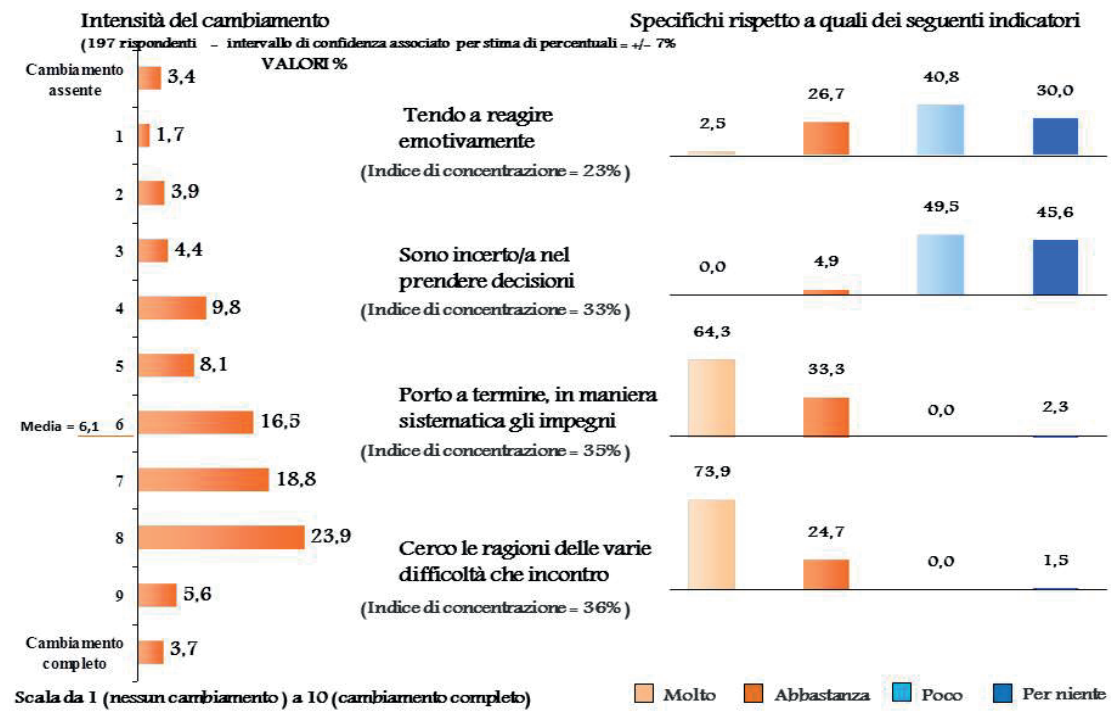

Figura 7. - Auto-percezione del cambiamento nello stile relazionale adottato a seguito dei tre anni di formazione e-learning in Scienze dell'Educazione, registrata da 197 docenti al Questionario CDVR.

\section{Cambiamento stile relazionale nel triennio}

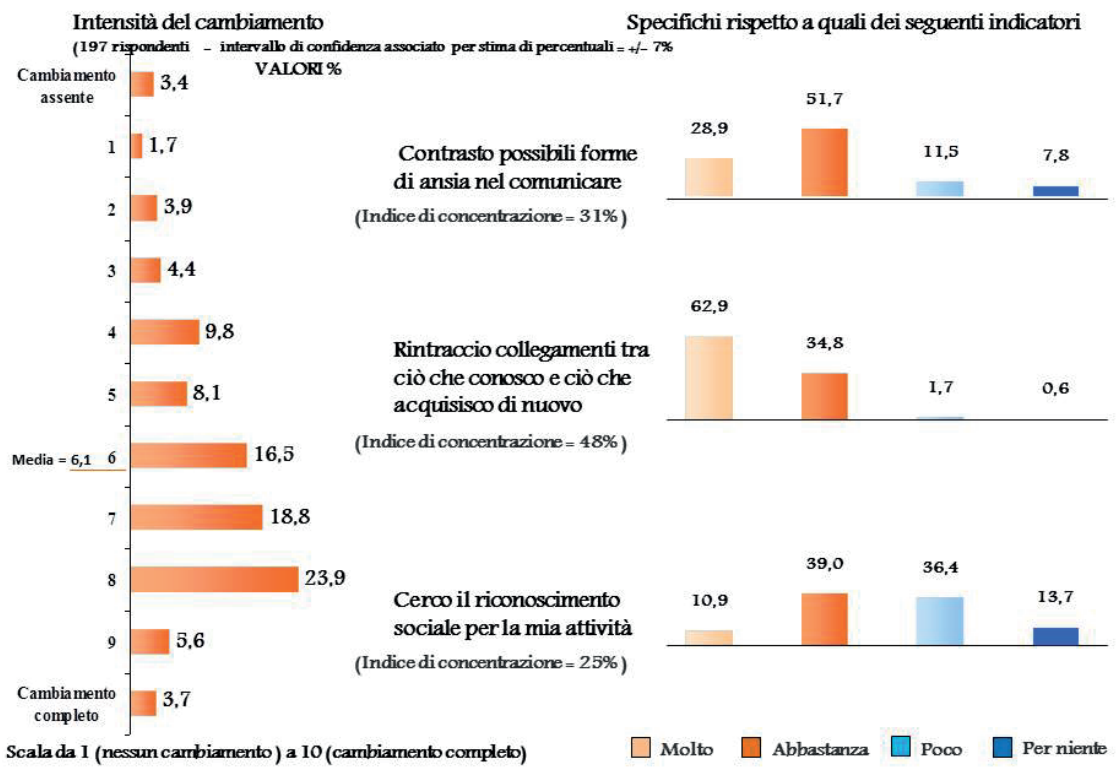

Figura 8. - Auto-percezione del cambiamento nello stile relazionale adottato a seguito dei tre anni di formazione e-learning in Scienze dell'Educazione, registrata da 197 docenti al Questionario CDVR. 


\section{Cambiamento stile relazionale nel triennio}

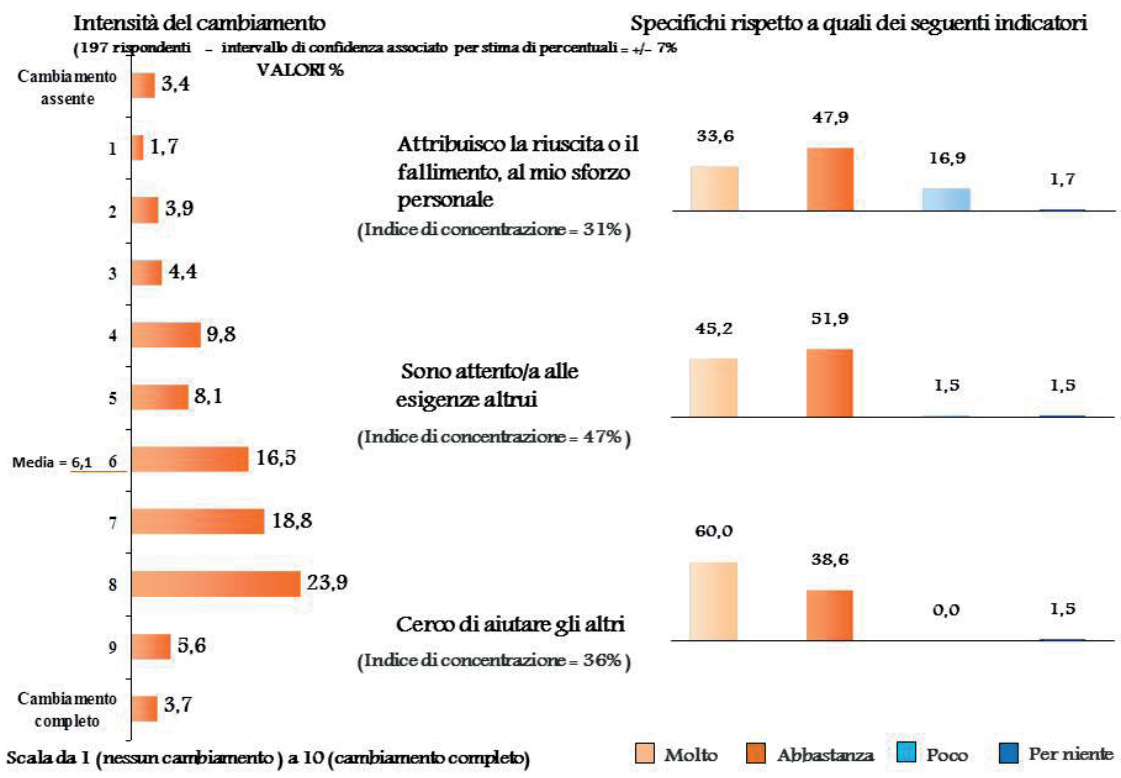

Figura 9. - Auto-percezione del cambiamento nello stile relazionale adottato a seguito dei tre anni di formazione e-learning in Scienze dell'Educazione, registrata da 197 docenti al Questionario CDVR.

Conoscere meglio chi si ha di fronte e comunicare meglio con chi si ha di fronte (grazie a migliori strategie didattico-valutative e a più approfondite conoscenze psicologiche), infatti, porta una speciale ricaduta sulla capacità di interagire e di sviluppare un atteggiamento positivo nell'ambito della relazione educativa, atteggiamento funzionale al potenziamento dei processi di apprendimento e quindi al raggiungimento degli esiti formativi perseguiti.

Le conoscenze acquisite hanno, dunque, a loro volta promosso un cambiamento nel modo in cui i docenti si relazionano agli studenti, ossia un cambiamento nelle competenze relazionali, nonché nella qualità della relazione educativa. Questo rinnovato profilo dell'identità professionale, e delle competenze ad esso correlate, come rilevato attraverso il Questionario CDVR, potrà a nostro avviso rappresentare la condizione di base per attuare una didattica innovativa flessibile ed integrata in grado di promuovere il più ampio successo formativo degli allievi (cfr. PRIN, 2010/2011). 


\section{RIFERIMENTI BIBLIOGRAFICI}

Ajello, A. M. (a cura di). (2002). La competenza. Bologna: il Mulino.

Altet, M. (2010). La relation dialectique entre pratique et théorie dans une formation professionnalisante des enseignants en IUFM: d'une opposition à une nécessaire articulation. Education Sciences \& Society, 1(1), 117-141.

Andreani Dentici, O. (2001). Intelligenza e creatività. Roma: Carocci.

Argyris, C., \& Schön, D. A. (1978). Organizational learning: A theory of action perspective. Cambridge, MA: Addison-Wesley.

Baldacci, M. (2010). Curricolo e competenze. Milano: Mondadori.

Bandura, A. (2000). Autoefficacia. Teoria e applicazioni. Trento: Erickson.

Biasi, V. (2006). Il conflitto psichico. Analisi fenomenologiche e verifiche sperimentali. Roma: Monolite.

Biasi, V., \& Bonaiuto, P. (2004). Dynamics of creativity performances under short term stress (or comfort) conditions. In J. P. Fròis, P. Andrade, \& J. F. Marques (Eds.), Art and science (pp. 411-415). Proceedings of the XVIII Congress of the International Association of Empirical Aesthetics. Lisbon: IAEA.

Biasi, V., \& Bonaiuto, P. (2007). Effetti dello stress sperimentale su creatività e percezione fisionomica. In A. Fusco \& R. Tomassoni (a cura di), I processi creativi artistici e letterari (pp. 80-111). Milano: FrancoAngeli.

Biasi, V., \& Bonaiuto, P. (2011). Creative processes and poetry production. The contribution of experimental psychology. In A. Fusco \& R. Tomassoni (a cura di), Analogie e diversità tra cultura scandinava e cultura italiana (pp. 81-112). Atti del Convegno Internazionale, Cassino, 9-12 Maggio. Roma: Teseo.

Biasi, V., Chiappetta Cajola, L., \& Bonaiuto, P. (2010). Valutare la formazione degli schemi mentali nei disturbi dell'apprendimento / Evaluating the formation of mental schemata in learning disorders. Journal of Educational, Cultural and Psychological Studies, 2(1), 117-138.

Bonaiuto, P., Biasi, V., Giannini, A. M., Bonaiuto, M., \& Bartoli, G. (1992). Stress, comfort and self-appraisal: A panoramic investigation of the dynamics of cognitive processes. In D. G. Forgays, T. Sosnowski, \& K. Wrzesniewski (Eds.), Anxiety: Recent developments in cognitive, psychophysiologycal and health research (pp. 75-107). Washington: Hemisphere.

Bonaiuto, P., Giannini, A. M., \& Biasi, V. (2003). Perception theories and the environmental experience. In M. Bonnes, T. Lee, \& M. Bonaiuto (Eds.), Psychological theories for enviromental issues (pp. 95-136). Aldershot: Ashgate.

Calvani, A. (2000). L'impatto dei nuovi media nella scuola; verso una saggezza tecnologica. Convegno Nazionale FIDAE, Roma. http://www.scform.unifi.it/lte.

Carr, W., \& Kemmis, S. (1986). Becoming critical: Education, knowledge and action research. London: Falmer Press. 
Cesa-Bianchi, M., \& Antonietti, A. (2003). Creatività nella vita e nella scuola. Milano: Mondatori.

Ciraci, A. M. (2008). E-learning ed equità. Didattica online e nuove opportunità formative. Roma: Anicia.

Ciraci, A. M. (2009). La formazione universitaria a distanza degli insegnanti: ruolo dell'autovalutazione e ricadute delle competenze acquisite sull'attività professionale. In G. Domenici (a cura di), Valutazione a autovalutazione per la qualificazione dei processi formativi e-learning (pp. 93-112). Lecce: Pensa MultiMedia.

Ciraci, A. M. (2013). La sfida delle competenze per una scuola inclusiva. In L. Chiappetta Cajola \& A. M. Ciraci, Didattica inclusiva. Quali competenze per gli insegnanti? Parte seconda (pp. 125-218). Roma: Armando.

Ciraci, A. M. (2014). La formazione universitaria degli insegnanti «in servizio»: modalità didattiche e ricadute professionali. L'esperienza dell'Università Roma Tre. MeTis, 4(1). http://metis.progedit.com/.

Ciraci, A. M., \& Capogna, S. (2005). Certificazione delle competenze e strategie didattiche. Opportunità formative per l'equità sociale. Roma: Monolite.

Clarke, B., \& Chambers, P. (Eds.). (2005). Reflective practices: Mechanisms for learning. Worcester, UK: College Worcester.

Commissione Europea (2007). Comunicazione della Commissione al Parlamento europeo e al Consiglio. Improving the Quality of Teacher Education. COM (2007) 392, Bruxelles, 3 August. http://ec.europa.eu/education/com392_en.pdf.

Csikszentmihalyi, M. (1997). Creativity: Flow and the psychology of discovery and invention. New York: Harper Collins.

Darling-Hammond, L. et al. (2005). Does teacher preparation matter? Evidence about teacher certification, teach for America, and teacher effectiveness. Education Policy Analysis. Archives, 13(42) 16-20.

Deci, E. L., \& Moller, A. C. (2007). The concept of competence. In J. Elliot \& C. S. Dweck (Eds.). Handbook of motivation and competence (pp. 579-597). New York: Guilford.

Demetrio, D. (1991). Tornare a crescere. L'età adulta tra persistenze e cambiamenti. Milano: Guerini e Associati.

Dewey, J. (1933). How we think. Boston: Heath.

Domenici, G. (1991). Gli strumenti della valutazione. Napoli: Tecnodid.

Domenici, G. (a cura di). (2000). La valutazione come risorsa. Napoli: Tecnodid.

Domenici, G. (2001). Manuale della valutazione scolastica. Bari - Roma: Laterza.

Domenici, G. (a cura di). (2005). Le prove semistrutturate di verifica degli apprendimenti. Torino: Utet.

Domenici, G. (a cura di). (2009a). La valutazione come risorsa. Napoli: Tecnodid. 
Domenici, G. (a cura di). (2009b). Valutazione e autovalutazione per la qualificazione dei processi formativi e-learning. Lecce: Pensa MultiMedia.

Domenici, G., Biasi, V., \& Ciraci, A. M. (2014). Evaluation of teaching and relational competencies for a flexible integrated didactic strategy: The CDVR Questionnaire. In Memorias (pp. 3011-3020). 9th International Congress of higher education University 2014, Havana, 10-14 February. Havana: UNIVERSIDAD 2014.

Domenici, G., Margottini, M., \& Cajola, L. (2006). Competenze informatiche pregresse e carriera universitaria degli studenti del CdL in Scienze dell'Educazione - FAD dell'Università Roma Tre. In G. Domenici (a cura di), La ricerca didattica per la formazione degli insegnanti (pp. 205-220). Atti del V Congresso Scientifico SIRD. Roma: Monolite.

Eysenck, H. J. (1993). Creativity and personality: Suggestions for a theory. Psychological Inquiry, 4, 147-178.

Falat, M. (2000). Creativity as a predictor of "good» coping? Studia Psychologica, 42(4), 317-324.

Furlong, J., Barton, L., Miles, S., Whiting, C., \& Whitty, G. (2000). Teacher education in transition. Re-forming professionalism? Buckimgham: Open University Press.

Galliani, L. (2004). La scuola in rete. Roma - Bari, Laterza.

Garroni, E. (2010). Creatività. Macerata: Quodlibet.

Ghaye, A., \& Ghaye, K. (1998). Teaching and learning through critical reflective practice. London: David Fulton.

Guilford, J. P. (1967). The nature of human intelligence. New York: McGraw-Hill.

Hinton, B. L. (1971). Personality factors and resistance to the effects of frustrations on creative problem-solving performance. Journal of Creative Behavior, 5(4), 267-269.

Kohler, W. (1925). The mentality of apes. New York: Harcourt - Brace (trad. it., Firenze: Universitaria, 1958).

Korthagen, F. A. (Ed.). (2001). Linking practice and learning. Mahwah, NJ: Lawrence Erlbaum Associates Publishers.

Krampen, G. (1997). Promotion of creativity (divergent productions) and convergent productions by systematic relaxation exercises: Empirical evidence from five experimental studies with children, young adults and elderly. European Journal of Personality, 11, 83-99.

Le Boterf, G. (2000). Construire les compétences individuelles et collectives. Paris: Éditions d'Organisation.

Legrenzi, P. (2005). Creatività e innovazione. Bologna: il Mulino.

Leith, G. (1972). The relationships between intelligence, personality and creativity under two conditions of stress. British Journal of Educational Psychology, 42(3), 240-247. 
Maccario, D. (2006). Insegnare per competenze. Torino: SEI.

Margottini, M. (2009). Rilevazione e analisi delle competenze digitali nella formazione on line. In G. Domenici \& R. Semeraro (a cura di), Le nuove sfide della ricerca didattica tra saperi, comunità sociali e culture (pp. 335-344). Atti del VI Congresso Scientifico SIRD. Roma: Monolite.

Mezirow, J. (1991). Transformative dimensions of adult learning. San Francisco: JosseyBass.

Mortari, L. (2003). Apprendere dall'esperienza. Il pensare riflessivo nella formazione. Roma: Carocci.

OECD - CERI (1994). Quality in teaching. Paris: OECD. http://www.oecd.org/.

OECD - CERI (1998). Making the curriculum work. Paris: OECD. http://www.oecd. org/.

OECD (2005). Teachers matter: Attracting, developing and retaining effective teachers. Paris: OECD. http://www.oecd.org/.

OECD (2009). Creating effective teaching and learning environments. First results from TALIS. Paris: OECD. http://www.oecd.org/.

OECD (2014). TALIS 2013 results: An international perspective on teaching and learning. Paris: OECD. http://dx.doi.org/10.1787/9789264196261-en.

Parker, S. (1997). Reflective teaching in a post-modern world. Buckingham: Open University Press.

Pellerey, M. (1996). Questionario sulle strategie d'apprendimento. Roma: LAS.

Pellerey, M. (2010). Ripensare le competenze. Napoli: Tecnodid.

Pellerey, M. (2011). Competenza. Education Science \& Society, 1, 173-179.

Perrenoud, Ph. (1996). Enseigner: agir dans l'urgence, décider dans l'incertitude. Paris: ESF.

Perrenoud, Ph. (1998). Construire des compétences dès l'école. Paris: ESF.

Perrenoud, Ph. (2001). Développer la pratique réflexive dans le métier d'enseignant. Professionnalisation et raison pédagogique. Paris: ESF.

Perrenoud, Ph. (2002). Dieci nuove competenze per insegnare. Invito al viaggio. Roma: Anicia.

Piaget, J. (1974). Réussir et comprendre. Paris: PUF.

PRIN - Programmi di ricerca di interesse nazionale (2010/2011). Bando anno 2010/2011. Area 11: Successo formativo, inclusione e coesione sociale: strategie innovative, ICT e modelli valutativi. Coordinatore scientifico nazionale G. Domenici (http://prin.cineca.it) dell’Università «Roma Tre».

Rey, B., Carette, V., Defrance, A., \& Kahn, S. (2003). Les compétences à l'école. Bruxelles: De Boeck.

Rivkin, S. G., Hanushek, E. A., \& Kain, J. F. (2005). Teachers, schools, and academic achievement. Econometrica, 73(2), 417-458. 
Runco, M. A., \& Albert, R. I. (Eds.). (1990). Theories of Creativity. Newbury Park, CA: Sage.

Runco, M. A., \& Richards, R. (1997). Eminent creativity, everyday creativity, and health. Greenwich, CT: Ablex.

Schön, D. A. (1983). The reflexive practitioner. New York: Basic Books.

Shaheen, R. (2010). Creativity and education. Creative Education, 1(3), 166-169.

Simonton, D. K. (1997). Genius and creativity. Selected papers. Greenwich, CT: Ablex.

Sternberg, R. J. (Ed.). (1999). Handbook of creativity. New York: Cambridge University Press.

Sternberg, R. J., \& Lubart, T. I. (1999). The concept of creativity: Prospects and paradigms. In Sternberg, 1999 (pp. 3-16).

Torrance, E. P. (1966). Torrance test of creative thinking. Directions manual and scoring guide. Lexington: Personnel Press.

Van Manen, M. (1993). The tact of teaching: The meaning of pedagogical thoughtfulness. Ann Arbor, MI: The Althouse Press.

Weiner, B. (1972). Theory of motivation: From mechanism to cognition. Chicago: RandMcNally.

Zeichner, K. M., \& Liston, D. (1996). Reflective teaching: An introduction. Mahwah, NJ: Lawrence Erlbaum Associates Publishers.

\section{RiassunTO}

Il presente contributo concerne la rilevazione delle competenze professionali degli insegnanti e la determinazione delle modalità didattico-formative più idonee per la loro acquisizione. Si tratta di una ricerca empirica condotta nel 2012/2013 da Domenici, Biasi e Ciraci, per il Dipartimento di Scienze della Formazione dell'Università "Roma Tre», che ha coinvolto 287 insegnanti in servizio nelle scuole primarie della Regione Lazio, laureatisi presso il "Corso di Laurea in Scienze dell'Educazione in modalità FAD su piattaforma e-learning". Si indagano le ricadute professionali ottenute al termine del percorso formativo, attraverso il Questionario online CDVR per la valutazione delle competenze didattico-valutative e relazionali. I risultati hanno mostrato che il suddetto percorso formativo universitario, condotto in modalità e-learning attraverso una didattica attiva basata sull'auto-valutazione e su innovative strategie di simulazione di contesti, ha prodotto un incremento della percezione delle proprie competenze didattico-valutative e relazionali, sviluppando nel docente la percezione della capacità di comprendere gli altri, ed una maggiore flessibilità mentale o creatività. Tali modalità didattiche attive, grazie alle nuove tecnologie, hanno promosso lo sviluppo di atteggiamenti alla base delle "competenze professionali» dell'insegnante esperto, mentalmente flessibile, creativo, nel trovare soluzioni originali ai molteplici problemi educativi da affrontare. Conoscere meglio chi si ha di fronte 
e comunicare meglio (grazie a migliori strategie didattico-valutative e a più approfondite conoscenze psicologiche), ha portato una speciale ricaduta sulla capacità di interagire e di sviluppare un atteggiamento positivo nell'ambito della relazione educativa, atteggiamento funzionale al potenziamento dei processi di apprendimento e quindi al raggiungimento degli esiti formativi perseguiti.

Parole chiave: Competenze didattiche e valutative, Competenze relazionali, Creatività, e-learning, Formazione degli insegnanti, Valutazione. 